\title{
Chemical Constituents of Morinda citrifolia Roots Exhibit Hypoglycemic Effects in Streptozotocin-Induced Diabetic Mice
}

\author{
Kohei Kamiya, * Wakako Hamabe, Sachiko Harada, Rie Murakami, Shogo Tokuyama, and \\ Toshiko SATAKE \\ Faculty of Pharmaceutical Sciences, Kobe Gakuin University; 1-1-3 Minatojima, Chuo-ku, Kobe 650-8586, Japan. \\ Received January 9, 2008; accepted February 21, 2008; published online February 25, 2008
}

\begin{abstract}
The hypoglycemic effects of the chemical constituents of Morinda citrifolia roots was evaluated in streptozotocin (STZ)-induced diabetic mice. The $\mathrm{CHCl}_{3}$, EtOAc, $n$-BuOH and $\mathrm{H}_{2} \mathrm{O}$ soluble phases of the $\mathrm{MeOH}$ extract of $M$. citrifolia roots were administrated orally to STZ-induced diabetic mice. Only the $n$-BuOH soluble phase showed a significant reduction of the blood glucose levels. From the biologically active $n$-BuOH soluble phase, two iridoids and three anthraquinones were isolated as main constituents. These compounds were identified by spectroscopic analysis to be deacetylasperulosidic acid (1), asperulosidic acid (2), damnacanthol-3- $O$ - $\beta$-Dprimeveroside (3), lucidin 3- $O$ - $\beta$-D-primeveroside (4) and morindone-6- $O$ - $\beta$-D-primeveroside (5). 3 and 4 exhibited the hypoglycemic effects, which were anthraquinones with no substituents in one aromatic ring.
\end{abstract}

Key words Morinda citrifolia; streptozotocin; hypoglycemic effect; anthraquinone glycoside

Morinda citrifolia L. (Rubiaceae), known as "noni," is a small tree that grows widely across Polynesia. In Japan, it is called "Yaeyama-aoki" and grows in Okinawa Prefecture. In Polynesia, M. citrifolia has traditionally been used for antibacterial, antiviral, antifungal, antitumor, antihelmin, analgesic, hypotensive, anti-inflammatory, and immune enhancing effects. ${ }^{1)}$ Furthermore, "noni juice," which is made from the fruit of this plant, is today widely drunk for its purported ability to prevent lifestyle-related diseases such as diabetes, hypertension, and cardiopathy and cerebral apoplexy caused by arteriosclerosis. In our previous study, six lignanes which inhibit the LDL oxidation process leading to arteriosclerosis were isolated from the fruit of $M$. citrifolia. $^{2)}$ Other pharmacological studies of $M$. citrifolia reported that iridoids isolated from the leaves of this plant inhibited UVB-induced Activator Protein-1 activity ${ }^{3}$ and the fruit of this plant prolonged the life span of C57BL/6 mice infected with Lewis lung carcinoma. ${ }^{4)}$ The above reports suggest that $M$. citrifolia leaves and fruits exhibit some anticancer effects. In addition, M. citrifolia root exhibited an analgesic effect. ${ }^{5}$ )

During the course of our search for natural products capable of preventing lifestyle-related diseases, we have investigated the ability of the chemical constituents of $M$. citrifolia roots to improve hyperglycemia or diabetes. In this study, we used streptozotocin (STZ)-induced diabetic mice, which is known as an insulin-deficiency model, to demonstrate the effects of $M$. citrifolia roots on blood glucose levels.

\section{MATERIALS AND METHODS}

Plant Material The roots of $M$. citrifolia were collected at Okinawa in August 2004. A voucher specimen has been retained in our laboratory.

Animals Male ddY mice weighing $c a$. $30 \mathrm{~g}$ (5 weeks old) were obtained from SLC (Osaka, Japan). The animals were housed at a temperature of $23-24^{\circ} \mathrm{C}$ with a $12 \mathrm{~h}$ light-dark cycle (lights on 8:00 a.m. to 8:00 p.m.). Food and water were available ad libitum. The present study was conducted in accordance with the Guiding Principles for the Care and Use of Laboratory Animals, adopted by the Japan- ese Pharmacological Society.

Streptozotocin (STZ)-Induced Diabetic Model Mice were injected intraperitoneally (i.p.) with $200 \mathrm{mg} / \mathrm{kg} \mathrm{STZ}$ (Sigma-Aldrich, St. Louis, MO, U.S.A.) prepared in $0.1 \mathrm{~N}$ citrate buffer adjusted to $\mathrm{pH} 4.3$. Only mice with plasma glucose levels greater than $400 \mathrm{mg} / \mathrm{dl}$ were considered as diabetic.

Measurement of Blood Glucose Levels The plasma glucose levels in the mice were measured using the Glucose Pilot (Aventir Biotech, CA, U.S.A.) in blood samples obtained from tail vein at 0 or $5 \mathrm{~h}$ after a single administration of $\mathrm{MeOH}$ extract and $\mathrm{CHCl}_{3}$, EtOAc, $n-\mathrm{BuOH}$ and $\mathrm{H}_{2} \mathrm{O}$ soluble phases of $M$. citrifolia roots (each at $3 \mathrm{~g} / \mathrm{kg}$, oral route, p.o.).

General Procedures Optical rotations were measured using a Jasco DIP-1000 digital polarimeter. IR and UV spectra were measured on a Shimadzu FT-IR 8300 infrared spectrometer and a Hitachi U-3000 spectrometer, respectively. NMR spectra were recorded in $\mathrm{CD}_{3} \mathrm{OD}$ and DMSO- $d_{6}$ on a Bruker DPX-400 instrument. TLC was performed on Merck

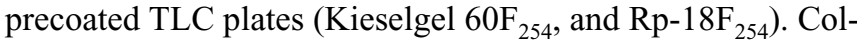
umn chromatography was conducted with Kieselgel 60 (70230 mesh, Merck) and Sephadex LH-20 (Pharmacia). Medium pressure liquid chromatography (MPLC, BUCHI Pump Module C-605 and Manager C-615) was carried out on a CIG column.

Extraction and Isolation The dried roots of $M$. citrifolia $(4.7 \mathrm{~kg})$ were extracted with $\mathrm{MeOH}(51 \times 10)$ for $6 \mathrm{~h}$ at $60{ }^{\circ} \mathrm{C}$. The combined $\mathrm{MeOH}$ was evaporated under reduced pressure to give the $\mathrm{MeOH}$ extract $(705 \mathrm{~g})$, which was suspended in $\mathrm{H}_{2} \mathrm{O}: \mathrm{MeOH}(3: 1,3.61)$ and then was sequentially partitioned with $\mathrm{CHCl}_{3}$, EtOAc and $n-\mathrm{BuOH}$ (each $3.61 \times 3$ ). Each solvent was removed under reduced pressure to yield $\mathrm{CHCl}_{3}$ soluble phase (123 g), EtOAc soluble phase (13 g), $n$ $\mathrm{BuOH}$ soluble phase (149 g) and $\mathrm{H}_{2} \mathrm{O}$ soluble phase $(420 \mathrm{~g})$. The $n-\mathrm{BuOH}$ soluble phase was subject to column chromatography on Sephadex LH-20 (MeOH) to give fractions $1-4$. Fraction 2 was subjected repeatedly to further column chromatography on $\mathrm{SiO}_{2}\left(\right.$ EtOAc- $\mathrm{MeOH}-\mathrm{H}_{2} \mathrm{O}$ solvent system) and $\mathrm{Rp}-18\left(\mathrm{H}_{2} \mathrm{O}: \mathrm{MeOH}=70: 30\right)$ to furnish com- 
pounds $\mathbf{1}-\mathbf{5}$.

HPLC Analysis HPLC was performed with an ODS column (Atlantis $\mathrm{dC}_{18} 5 \mu \mathrm{M}, 4.6 \times 250 \mathrm{~mm}$, Waters). The detection wavelength was $254 \mathrm{~nm}$. Elution was carried out with $\mathrm{MeOH}-\mathrm{H}_{2} \mathrm{O}$ containing $0.1 \%$ phosphoric acid $(47.5: 52.5)$ at a flow rate of $1 \mathrm{ml} / \mathrm{min}$. Injection volume was $10 \mu \mathrm{l}$ $(1.0 \mathrm{mg} / \mathrm{ml}, n-\mathrm{BuOH}$ soluble phase in $\mathrm{MeOH})$.

Compound 1 (Deacetylasperulosidic Acid): Colorless amorphous powder, $[\alpha]_{\mathrm{D}}^{23}+22.4^{\circ}(c=0.13, \mathrm{MeOH})$; IR $v_{\max }^{\mathrm{KBr}}$ $\mathrm{cm}^{-1}: 3373,2928,1688,1680,1634,1420,1265,1200$, 1157, 1078, 1049, 991; UV $\lambda_{\max }^{\mathrm{MeO}} \mathrm{nm}(\log \varepsilon): 234$ (3.88), 200 (3.72); ${ }^{1} \mathrm{H}-\mathrm{NMR}\left(400 \mathrm{MHz}, \mathrm{CD}_{3} \mathrm{OD}\right)$ and ${ }^{13} \mathrm{C}-\mathrm{NMR}$ (100 MHz, $\left.\mathrm{CD}_{3} \mathrm{OD}\right)$ : Table 1.

Compound 2 (Asperulosidic Acid): Colorless amorphous powder, $[\alpha]_{\mathrm{D}}^{23}+21.4^{\circ}(c=0.14, \mathrm{MeOH})$; IR $v_{\max }^{\mathrm{KBr}} \mathrm{cm}^{-1}$ : $3400,2929,1720,1690,1635,1369,1252,1200,1158$, 1075, 1047, 949; UV $\lambda_{\max }^{\mathrm{MeOH}} \mathrm{nm}(\log \varepsilon): 233$ (3.80), 201 (3.86); ${ }^{1} \mathrm{H}-\mathrm{NMR} \quad\left(400 \mathrm{MHz}, \quad \mathrm{CD}_{3} \mathrm{OD}\right)$ and ${ }^{13} \mathrm{C}-\mathrm{NMR}$ (100 MHz, $\left.\mathrm{CD}_{3} \mathrm{OD}\right)$ : Table 1 .

Compound 3 (Damnacanthol-3- $O$ - $\beta$-D-primeveroside): Yellow needles, $[\alpha]_{\mathrm{D}}^{23}-128.62^{\circ}\left(c=0.13, \mathrm{MeOH} / \mathrm{H}_{2} \mathrm{O}=\right.$ $1: 1)$; IR $v_{\max }^{\mathrm{KBr}} \mathrm{cm}^{-1}: 3380,2883,1672,1580,1452,1410$, $1327,1300,1283,1070,1035,991 ; \mathrm{UV} \lambda_{\max }^{\mathrm{MeOH}} \mathrm{nm}(\log \varepsilon)$ : 266 (4.51), 201 (4.40); ${ }^{1} \mathrm{H}-\mathrm{NMR}$ (400 MHz, CD 3 OD) and ${ }^{13} \mathrm{C}$-NMR (100 MHz, $\left.\mathrm{CD}_{3} \mathrm{OD}\right)$ : Table 2.

Compound 4 (Lucidin 3-O- $\beta$-D-primeveroside): Yellow needles, $[\alpha]_{\mathrm{D}}^{23}-118.9^{\circ} \quad\left(c=0.11, \mathrm{MeOH} / \mathrm{H}_{2} \mathrm{O}=1: 1\right) ; \mathrm{IR}$ $v_{\max }^{\mathrm{KBr}} \mathrm{cm}^{-1}: 3379,2891,1672,1632,1591,1483,1420,1369$,
1333, 1296, 1075, 1043; UV $\lambda_{\max }^{\mathrm{MeOH}} \mathrm{nm}(\log \varepsilon): 266(4.45)$, 246 (4.42), 240 (4.40), 204 (4.52); ${ }^{1} \mathrm{H}-\mathrm{NMR} \quad(400 \mathrm{MHz}$, $\left.\mathrm{CD}_{3} \mathrm{OD}\right)$ and ${ }^{13} \mathrm{C}-\mathrm{NMR}\left(100 \mathrm{MHz}, \mathrm{CD}_{3} \mathrm{OD}\right)$ : Table 2.

Compound 5 (Morindone-6- $O-\beta$-D-primeveroside): Yellow

Table 1. ${ }^{1} \mathrm{H}$ - and ${ }^{13} \mathrm{C}$-NMR Spectral Data for Compounds $\mathbf{1}$ and $\mathbf{2}$ in $\mathrm{CD}_{3} \mathrm{OD}$

\begin{tabular}{|c|c|c|c|c|}
\hline \multirow{2}{*}{ No. } & \multicolumn{2}{|r|}{1} & \multicolumn{2}{|r|}{2} \\
\hline & ${ }^{13} \mathrm{C}$ & ${ }^{1} \mathrm{H}$ & ${ }^{13} \mathrm{C}$ & ${ }^{1} \mathrm{H}$ \\
\hline 1 & 101.48 & $5.06(\mathrm{~d}, 9.0)$ & 101.20 & $5.06(\mathrm{~d}, 9.0)$ \\
\hline 3 & 155.40 & $7.66(\mathrm{~d}, 0.9)$ & 155.47 & 7.67 (brs) \\
\hline 4 & 108.46 & & 108.04 & \\
\hline 5 & 42.70 & $3.01(\mathrm{ddd}, 7.1,5.8,0.9)$ & 42.24 & $3.02($ brt, 6.4$)$ \\
\hline 6 & 75.28 & $4.82(\mathrm{dd}, 5.8,1.6)$ & 72.73 & $3.28(\mathrm{~d}, 8.7)$ \\
\hline 7 & 129.89 & $6.02(\mathrm{~d}, 1.6)$ & 131.78 & $6.02(\mathrm{brs})$ \\
\hline 8 & 151.42 & & 145.72 & \\
\hline 9 & 45.81 & $2.56(\mathrm{dd}, 9.0,7.1)$ & 46.07 & 2.63 (brt, 8.2) \\
\hline 10 & 61.67 & 4.46 (br d, 15.6) & 63.67 & 4.95 (br d, 15.1) \\
\hline & & $4.21(\mathrm{brd}, 15.6)$ & & $4.83($ br d, 15.1) \\
\hline 11 & 170.86 & & 170.60 & \\
\hline $\mathrm{COCH}_{3}$ & & & 20.78 & $2.10(\mathrm{~s})$ \\
\hline$\underline{\mathrm{COCH}}_{3}$ & & & 172.55 & \\
\hline $1^{\prime}$ & 100.40 & $4.73(\mathrm{~d}, 7.9)$ & 100.42 & $4.75(\mathrm{~d}, 7.8)$ \\
\hline $2^{\prime}$ & 74.92 & & 75.23 & \\
\hline $3^{\prime}$ & 78.41 & & 78.21 & \\
\hline $4^{\prime}$ & 71.58 & $3.23-3.87$ & 71.35 & $3.25-3.88$ \\
\hline $5^{\prime}$ & 77.75 & & 77.63 & \\
\hline $6^{\prime}$ & 62.79 & & 62.81 & \\
\hline
\end{tabular}

Coupling patterns and coupling constants $(J)$ in $\mathrm{Hz}$ are given in parentheses.

Table 2. ${ }^{1} \mathrm{H}$ - and ${ }^{13} \mathrm{C}-\mathrm{NMR}$ Spectral Data for Compounds $\mathbf{3}-\mathbf{5}$ in DMSO- $d_{6}$

\begin{tabular}{|c|c|c|c|c|c|c|}
\hline \multirow{2}{*}{ No. } & \multicolumn{2}{|r|}{3} & \multicolumn{2}{|r|}{4} & \multicolumn{2}{|c|}{5} \\
\hline & ${ }^{13} \mathrm{C}$ & ${ }^{1} \mathrm{H}$ & ${ }^{13} \mathrm{C}$ & ${ }^{1} \mathrm{H}$ & ${ }^{13} \mathrm{C}$ & ${ }^{1} \mathrm{H}$ \\
\hline 1 & 160.74 & & 161.88 & & 160.18 & \\
\hline $1-\mathrm{OH}$ & & & & $12.92(\mathrm{~s})$ & & $12.92(\mathrm{~s})$ \\
\hline 2 & 131.64 & & 123.66 & & 135.07 & \\
\hline 3 & 160.68 & & 162.03 & & 137.03 & $7.50(\mathrm{~d}, 7.8)$ \\
\hline 4 & 109.19 & $7.75(\mathrm{~s})$ & 106.43 & $7.37(\mathrm{~s})$ & 118.60 & $7.45(\mathrm{~d}, 7.8)$ \\
\hline $4 a$ & 135.75 & & 133.65 & & 130.54 & \\
\hline 5 & 126.30 & $8.14(\mathrm{dd}, 7.5,1.3)$ & 126.88 & $8.06(\mathrm{~m})$ & 152.06 & \\
\hline $5-\mathrm{OH}$ & & & & & & $12.70(\mathrm{~s})$ \\
\hline 6 & 133.65 & $7.86(\mathrm{td}, 7.5,1.3)$ & 134.87 & $7.84(\mathrm{~m})$ & 151.64 & \\
\hline 7 & 134.70 & $7.91(\mathrm{td}, 7.5,1.3)$ & 134.73 & $7.84(\mathrm{~m})$ & 120.52 & $7.54(\mathrm{~d}, 8.6)$ \\
\hline 8 & 126.72 & $8.16(\mathrm{dd}, 7.5,1.3)$ & 126.46 & $8.06(\mathrm{~m})$ & 120.52 & $7.64(\mathrm{~d}, 8.6)$ \\
\hline $8 \mathrm{a}$ & 134.36 & & 132.59 & & 125.16 & \\
\hline 9 & 180.43 & & 186.95 & & 186.77 & \\
\hline $9 \mathrm{a}$ & 120.53 & & 111.30 & & 114.53 & \\
\hline 10 & 182.17 & & 181.32 & & 187.35 & \\
\hline $10 \mathrm{a}$ & 132.06 & & 132.74 & & 115.83 & \\
\hline \multirow[t]{2}{*}{11} & 51.99 & $4.68(\mathrm{dd}, 11.2,6.4)$ & 51.03 & $4.61(\mathrm{dd}, 11.2,5.7)$ & 15.74 & $2.18(\mathrm{~s})$ \\
\hline & & $4.61(\mathrm{dd}, 11.2,5.8)$ & & $4.52(\mathrm{dd}, 11.2,4.8)$ & & \\
\hline $\mathrm{OCH}_{3}$ & 62.74 & $3.89(\mathrm{~s})$ & & & & \\
\hline $1^{\prime}$ & 101.00 & $5.13(\mathrm{~d}, 7.2)$ & 100.89 & $5.09(\mathrm{~d}, 6.9)$ & 99.76 & $5.08(\mathrm{~d}, 7.2)$ \\
\hline $2^{\prime}$ & 73.29 & & 73.33 & & 73.05 & \\
\hline $3^{\prime}$ & 75.91 & & 75.96 & & 76.50 & \\
\hline $4^{\prime}$ & 69.53 & $3.01-4.01$ & 69.57 & $2.99-3.99$ & 69.60 & $2.93-4.00$ \\
\hline $5^{\prime}$ & 76.43 & & 76.48 & & 76.64 & \\
\hline $6^{\prime}$ & 68.06 & & 68.07 & & 68.38 & \\
\hline $1^{\prime \prime}$ & 104.09 & $4.18(\mathrm{~d}, 7.3)$ & 104.13 & $4.17(\mathrm{~d}, 7.3)$ & 104.05 & $4.15(\mathrm{~d}, 7.4)$ \\
\hline $2^{\prime \prime}$ & 73.33 & & 73.37 & & 73.48 & \\
\hline $3^{\prime \prime}$ & 75.74 & & 75.77 & & 76.08 & \\
\hline $4^{\prime \prime}$ & 69.24 & $3.01-4.01$ & 69.21 & $2.99-3.99$ & 69.53 & $2.93-4.00$ \\
\hline $5^{\prime \prime}$ & 65.66 & & 65.71 & & 65.61 & \\
\hline
\end{tabular}


needles, $[\alpha]_{\mathrm{D}}^{23}-71.3^{\circ}\left(c=0.3, \mathrm{MeOH} / \mathrm{H}_{2} \mathrm{O}=1: 1\right)$; IR $v_{\max }^{\mathrm{KBr}}$ $\mathrm{cm}^{-1}: 3350,1628,1603,1450,1423,1371,1261,1246$, 1057, 991; UV $\lambda_{\max }^{\mathrm{MeOH}} \mathrm{nm}(\log \varepsilon): 260$ (4.56), 230 (4.61); ${ }^{1} \mathrm{H}-\mathrm{NMR}\left(400 \mathrm{MHz}, \mathrm{CD}_{3} \mathrm{OD}\right)$ and ${ }^{13} \mathrm{C}-\mathrm{NMR}(100 \mathrm{MHz}$, $\left.\mathrm{CD}_{3} \mathrm{OD}\right)$ : Table 2 .

Statistical Analysis Data is presented as the mean with S.E.M. The statistical significance was assessed with a one way analysis of variance followed by the paired Student's $t$ test. The differences were regarded as significant when the $p$ value was less than 0.05 .

\section{RESULTS AND DISCUSSION}

The effects of the $\mathrm{MeOH}$ extract and $\mathrm{CHCl}_{3}$, EtOAc, $n$ $\mathrm{BuOH}$ and $\mathrm{H}_{2} \mathrm{O}$ soluble phases of $M$. citrifolia roots (each at $3 \mathrm{~g} / \mathrm{kg}$, p.o.) on blood glucose levels in STZ-induced diabetic mice are shown in Fig. 1. Only the $n$-BuOH soluble phase showed a significant reduction of the blood glucose levels at $5 \mathrm{~h}$ after administration $(303.9 \pm 25.6 \mathrm{mg} / \mathrm{dl})$ relative to those at $0 \mathrm{~h}$ after administration $(455.9 \pm 17 \mathrm{mg} / \mathrm{dl})$, while the $\mathrm{MeOH}$ extract and other soluble phases did not exhibit any hypoglycemic effect. As shown in Fig. 2, the dose-response curve of the hypoglycemic effect of the $n$-BuOH soluble phase in STZ-induced diabetic mice had a bell-shape with a maximum response at $1.0 \mathrm{~g} / \mathrm{kg}$, which decreased for doses over $3.0 \mathrm{~g} / \mathrm{kg}$. To elucidate the constituents of the biologically active $n$-BuOH soluble phase, HPLC analysis was performed

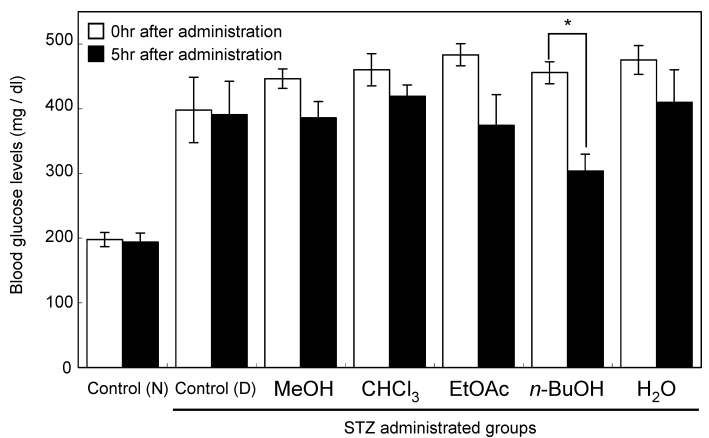

Fig. 1. Effects of the MeOH Extract and Each Soluble Phase of M. citrifolia Roots on Blood Glucose Levels in STZ-Induced Diabetic Mice

The blood glucose levels were measured at $0 \mathrm{~h}(\square)$ or $5 \mathrm{~h}(\boldsymbol{\square})$ after administration of each sample $(3 \mathrm{~g} / \mathrm{kg}$, p.o. $)$ as described in Materials and Methods. $* p<0.05$ vs. $0 \mathrm{~h}$. Control (N); non-treated normal mice; Control (D); STZ-induced diabetic mice.

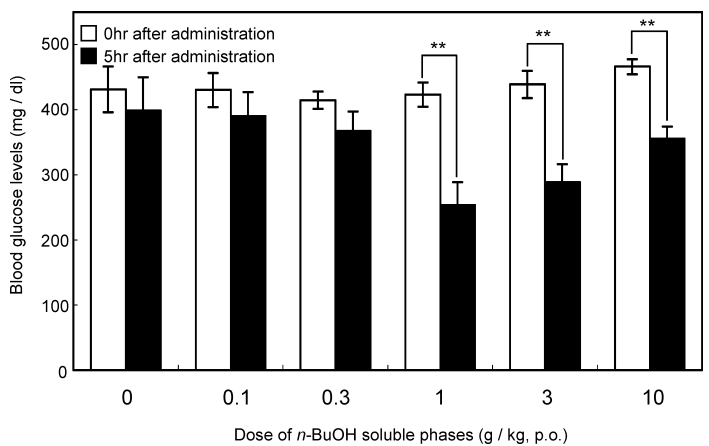

Fig. 2. The Dose-Dependent Hypoglycemic Effect of $n$-BuOH Soluble Phase of M. citrifolia Roots in STZ-Induced Diabetic Mice

The blood glucose levels were measured at $0 \mathrm{~h}(\square)$ or $5 \mathrm{~h}(\boldsymbol{\square})$ after administration of the $n$-BuOH soluble phase $(0,0.1,0.3,1,3,10 \mathrm{~g} / \mathrm{kg}$, p.o. $)$ as described in Materials and Methods. ** $p<0.01$ vs. $0 \mathrm{~h}$.
(Fig. 3), revealing five main peaks (compounds 1-5). Compounds $\mathbf{1}-\mathbf{5}$ were isolated by repeated column chromatography on Sephadex LH-20, $\mathrm{SiO}_{2}$ and Rp-18. Compounds 1 and 2 showed brilliant blue spots on TLC upon heating with $10 \%$ sulfuric acid, indicating them to be iridoid compounds. The chemical structures of $\mathbf{1}$ and $\mathbf{2}$ were confirmed to be deacetylasperulosidic acid and asperulosidic acid, respectively, by the comparison with published spectroscopic data. These compounds had previously been isolated from the fruits of the same plant. ${ }^{6)}$ Compounds 3, 4 and 5 were obtained as yellow needles and turned red on treatment with ammonia gas, indicating them to be anthraquinone compounds. The Chemical structures of 3, 4 and 5 were identified as damnacanthol-3-O$\beta$-D-primeveroside, ${ }^{7)}$ lucidin $3-O-\beta$-D-primeveroside (ophiohayatone-B $)^{8)}$ and morindone-6- $O$ - $\beta$-D-primeveroside, ${ }^{9)}$ respectively, by the comparison with published spectroscopic data.

Two iridoids and three anthraquinones were isolated from the biologically active $n$-BuOH soluble phase. The yields of $\mathbf{1}, \mathbf{2}, \mathbf{3}, \mathbf{4}$ and $\mathbf{5}$ were $0.18,0.49,0.27,0.20$ and $0.14 \%$ from the dried root, respectively. These compounds were tested for hypoglycemic effect in STZ-induced diabetic mice. Figure 5 shows the blood glucose levels 0 and $5 \mathrm{~h}$ after a single administration of each sample $(100 \mathrm{mg} / \mathrm{kg}$, p.o. $)$. Although the iridoids did not show a hypoglycemic effect, the two anthraquinones, 3 and 4 , showed a significant reduction of the blood glucose levels at $5 \mathrm{~h}$ after administration. However, another anthraquinone, $\mathbf{5}$ did not affect the blood glucose lev-

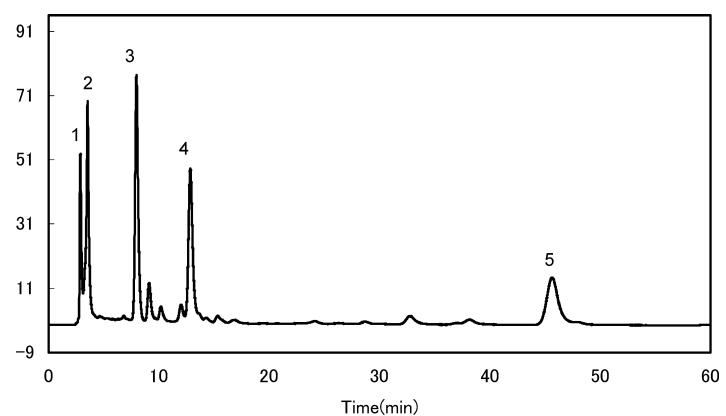

Fig. 3. HPLC Profile of $n$-BuOH Soluble Phase of $M$. citrifolia Roots

1 ; deacetylasperulosidic acid, 2 ; asperulosidic acid, 3 ; damnacanthol-3- $O-\beta$-Dprimeveroside, 4 ; lucidin $3-O-\beta$-D-primeveroside, 5 ; morindone-6- $O-\beta$-D-primeveroside.
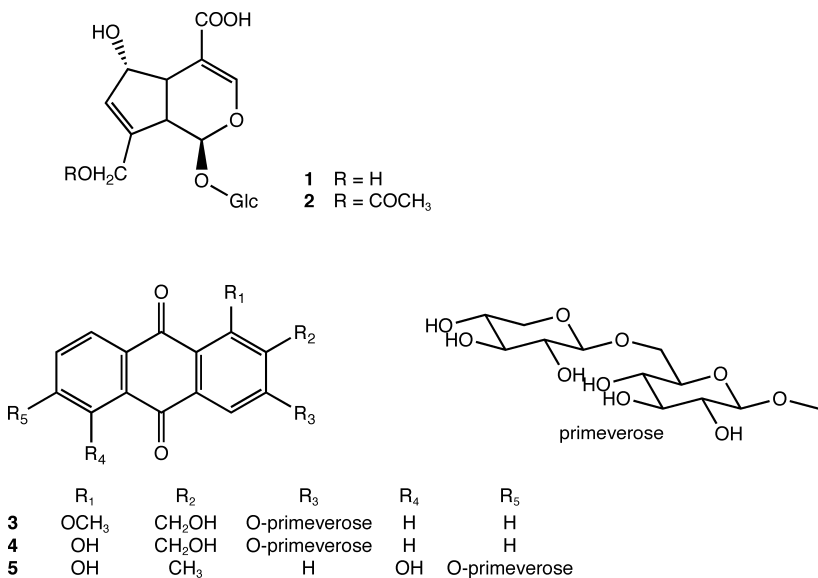

Fig. 4. Chemical Structures of Compounds $\mathbf{1}-\mathbf{5}$ Isolated from $n-\mathrm{BuOH}$ Soluble Phase of $M$. citrifolia Roots 


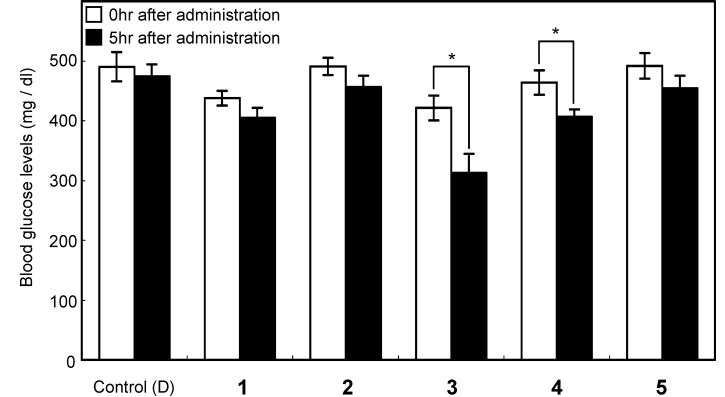

Fig. 5. The Hypoglycemic Effect of Compounds $\mathbf{1}-\mathbf{5}$ in STZ-Induced Diabetic Mice

The blood glucose levels were measured at $0 \mathrm{~h}(\square)$ or $5 \mathrm{~h}(\boldsymbol{\square})$ after administration of compounds $\mathbf{1}-\mathbf{5}$ (each $100 \mathrm{mg} / \mathrm{kg}$, p.o.) as described in Materials and Methods. $* p<0.05$ vs. $0 \mathrm{~h}$.

els. These finding indicate that the hypoglycemic effect of $M$. citrifolia roots in STZ-induced diabetic mice is due to the presence of anthraquinones. In particular, the anthraquinones that have no substituents on one aromatic ring seem to play an important role in the hypoglycemic effect of $M$. citrifolia, although the detailed mechanism of their hypoglycemic effect remains to be determined.

\section{REFERENCES}

1) Wang M. Y., West B. J., Jensen C. J., Nowicki D., Chen S., Palu A., Anderson G., Acta Pharmacol. Sin., 23, 1127-1141 (2002).

2) Kamiya K., Tanaka Y., Endang H., Umar M., Satake T., J. Agric. Food Chem., 52, 5843-5848 (2004).

3) Sang S., He K., Liu G., Zhu N., Wang M., Jhoo J. W., Zheng Q., Dong Z., Ghai G., Rosen R. T., Ho C. T., Tetrahedron Lett., 42, 1823-1825 (2001).

4) Hirazumi A., Furusawa E., Chou S. C., Hokama Y., Proc. West Pharmacol. Soc., 37, 145-146 (1994).

5) Younos C., Rolland A., Fleurentin J., Lanhers M. C., Misslin R., Mortier F., Planta Med., 56, 430- 434 (1990).

6) Kamiya K., Tanaka Y., Endang H., Umar M., Satake T., Chem. Pharm. Bull., 50, 1597-1599 (2003).

7) Li B., Zhang D. M., Luo Y. M., Chen X. G., Chem. Pharm. Bull., 54, 297-300 (2006).

8) Chan H. H., Li C. Y., Damu A. G., Wu T. S., Chem. Pharm. Bull., 53, 1232-1235 (2005).

9) Rao P. S., Reddy G. C. V., Indian J. Chem., Sec. B: Org. Chem. Incl. Med. Chem., 15B, 497-498 (1977). 